\title{
The Value of Predictive Nursing in Perioperative Period of Allogeneic Hematopoietic Stem Cell Transplantation
}

\author{
Yun Pan, Dongdong Chen* \\ Email address: \\ lajiao_420@163.com (Dongdong Chen) \\ ${ }^{*}$ Corresponding author
}

Department of Cardiology, The First Affiliated Hospital of Jinan University, Guangzhou, China

\section{To cite this article:}

Yun Pan, Dongdong Chen. The Value of Predictive Nursing in Perioperative Period of Allogeneic Hematopoietic Stem Cell Transplantation. Science Journal of Clinical Medicine. Vol. 9, No. 3, 2020, pp. 82-86. doi: 10.11648/j.sjcm.20200903.17

Received: September 3, 2020; Accepted: September 15, 2020; Published: September 28, 2020

\begin{abstract}
Background: Predictive nursing is a new concept proposed in recent years. Studies have shown that predictive care is effective in critically ill and surgical patients. However, the application and research of predictive nursing in patients with allogeneic hematopoietic stem cell transplantation (allo-HSCT) during perioperative period is still lacking. Objective: The value and clinical effect of predictive nursing in allo-HSCT during the perioperative period. Methods: Retrospective analysis was performed on 36 patients with hematological disease who had received allo-HSCT. Observation group A was the patient who received oral care, perineal care and perianal care during the transplantation period; group B was the patient who received only one of the three care items; group $\mathrm{C}$ was the patient who received no nursing intervention. And then the time of stem cell transplantation, the incidence of infection, and the cost of treatment were analyzed among the three groups. Results: Predictive nursing intervention can significantly shorten the transplantation time of granulocytes and megakaryocytes (A vs. C, $P=0.000, P=0.000$; B vs. C, $P=0.000, P=0.000$ ). Patients receiving oral care, perineal care and perianal care at the same time had a tendency to shorten the transplant time of stem cells and effectively reduce the incidence of respiratory and urinary tract infections compared with patients receiving only one nursing intervention $\left(\mathrm{X}^{2}=5.538, \mathrm{X}^{2}=5.924 ; P=0.019, P=0.05\right)$. And reduce the cost of treatment during transplantation (A vs. $\mathrm{C}, P=0.006$ ). Conclusions: In the perioperative period of allogeneic hematopoietic stem cells, various predictive nursing interventions can shorten the transplantation time of stem cells, reduce the incidence of infection, and thus reduce the economic burden of patients. It is a cheap and effective method and worthy of clinical application.
\end{abstract}

Keywords: Predictive Nursing, Allogeneic Hematopoietic Stem Cell Transplantation, Transplantation Time

\section{Introduction}

Allogeneic hematopoietic stem cell transplantation (allo-HSCT) is an adoptive immunotherapy method to treat hematologic malignancies, bone marrow hematopoietic diseases and genetic metabolic disorders $[1,2]$. The mechanism of treatment is that tumor cells, pathological hematopoietic cells and immune cells are cleared by preoperative super-dose chemotherapy or systemic extensive radiotherapy. Then the donor's healthy hematopoietic stem cells were implanted into the patient, so that the patient can restore normal hematopoietic and immune functions. This suggests that patients must experience a period of extreme immune deficiency prior to stem cell implantation survival and immune reconstitution, which studies have shown to last on average about 14 days [3]. During periods of immunodeficiency, infection is the most common complication. Once the infection occurs, it will be a fatal blow to the patient's life and economy. In clinical practice, the traditional preventative treatment including systemic drug bath and negative pressure aseptic laminar flow ward monitoring, both have been widely accepted. However, the prophylactic use of antibiotics is still controversial. In addition, predictive nursing is a new concept proposed in recent years, which refers to the simple way before the occurrence of adverse events to block the initiation of the event, so as to improve the prognosis of patients and reduce the cost of treatment $[4,5]$. Studies have shown that predictive care is effective in critically ill and surgical patients. However, the application and research of predictive nursing in patients with 
allo-HSCT during perioperative period is still lacking [6]. Therefore, the clinical data of 36 patients with hematological disease who had undergone allo-HSCT were retrospectively analyzed.

\section{Materials and Methods}

\subsection{Patients and Samples}

A retrospective analysis was performed on 36 patients with hematologic diseases treated with allo-HSCT from January 2016 to December 2019, including 28 cases of acute leukemia, 6 cases of myelodysplastic syndrome, and 2 cases of aplastic anemia. Inclusion criteria: patients' diseases were confirmed by bone marrow aspiration and bone marrow biopsy, no site infection was found from admission to allo-HSCT, and no antibiotic treatment was used. Exclusion criteria: patients with autoimmune related diseases, infection or adopted by antibiotic therapy, with difficulty in language communication or mental disorders, and Patients who cannot undergo allo-HSCT due to unsuitable match or economic conditions.

According to the purpose of retrospective analysis, patients were divided into group A, B and control group C. In group A, there were 10 males and 6 females, aged between 18 and 52 years old, with an average age of $(43 \pm 12)$ years old. In group $\mathrm{B}$, there were 4 males and 6 females, ranging in age from 16 to 56 years old, with an average age of $(44 \pm 11)$ years. In the control group, there were 6 males and 4 females, aged between 21 and 58 years old, with an average age of $(47 \pm 9)$ years old. There was no statistical difference in age, gender and other data of patients in each group.

\subsection{Groups}

Patients were divided into group A, B and C. Group A received oral care, perineal care and perianal care daily from 1 week before allo-HSCT to the perioperative period. Group B received only one of the three nursing interventions. Group $\mathrm{C}$ is the control group.

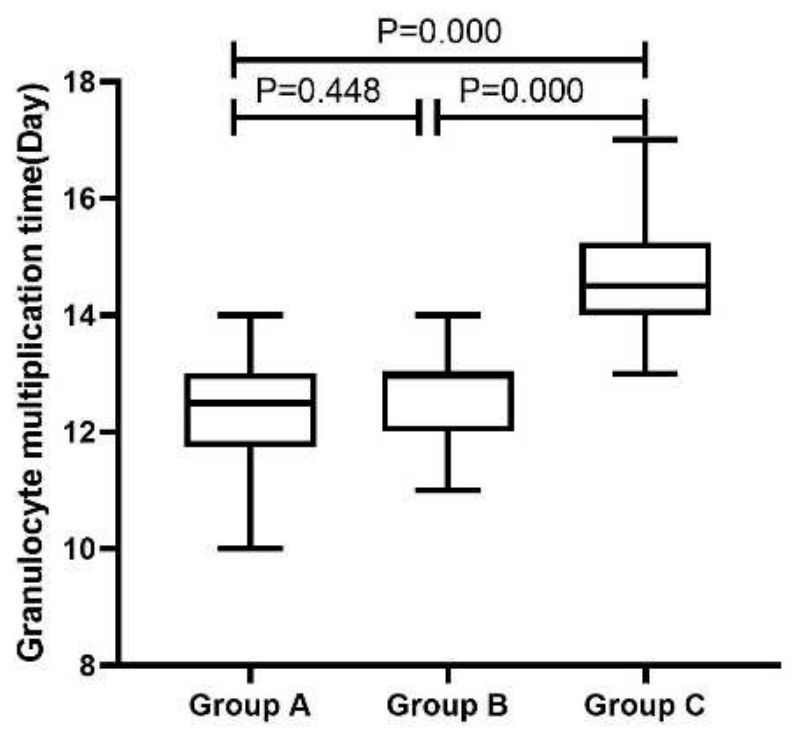

Figure 1. Comparison of granulocyte and platelet graft time in each group.

\subsection{Observation Indexes}

The first observation is the time of HSCT: from the day when stem cells were returned to the recipient to the successful time of stem cell transplantation, the criterion of granulocyte restoration was absolute value of neutrophils $>0.5 \times 10^{\wedge} 9 / \mathrm{L}$, and platelet $>20 \times 10^{\wedge} 9 / \mathrm{L}$ were the recovery criteria for megakaryocytes. Second is the incidence of perioperative infections (including respiratory, digestive and urinary tract infections). Third is perioperative hospitalization cost.

\subsection{Statistical Analysis}

SPSS20.0 statistical software was used for data processing. Chi-square test was used for counting data between groups. Two independent sample $\mathrm{T}$ tests were used for measurement data. Statistical significance was accepted at $p<0.05$.

\section{Results}

\subsection{Comparison of Successful Time of HSCT Between Group A, B and Control Group C}

The table 1 shows the transplantation time of granulocytes and platelets in each group. Compared with group $\mathrm{C}$, the transplantation time of granulocyte and platelet in group $\mathrm{A}$ and $\mathrm{B}$ were significantly reduced (A vs C, $P=0.000, P=0.000$; B vs C, $P=0.000, P=0.000)$, the results suggested that predictive nursing intervention in the perioperative period of HSCT could significantly shorten the transplantation time of granulocytes and megakaryocytes. In addition, further analysis found that patients receiving oral care, perineal care and perianal care at the same time had a tendency to shorten the transplant time of stem cells compared with patients receiving only one of the nursing interventions, but the difference was not statistically significant (A vs $\mathrm{B}, P=0.448$, $P=0.880$; figure 1 ).

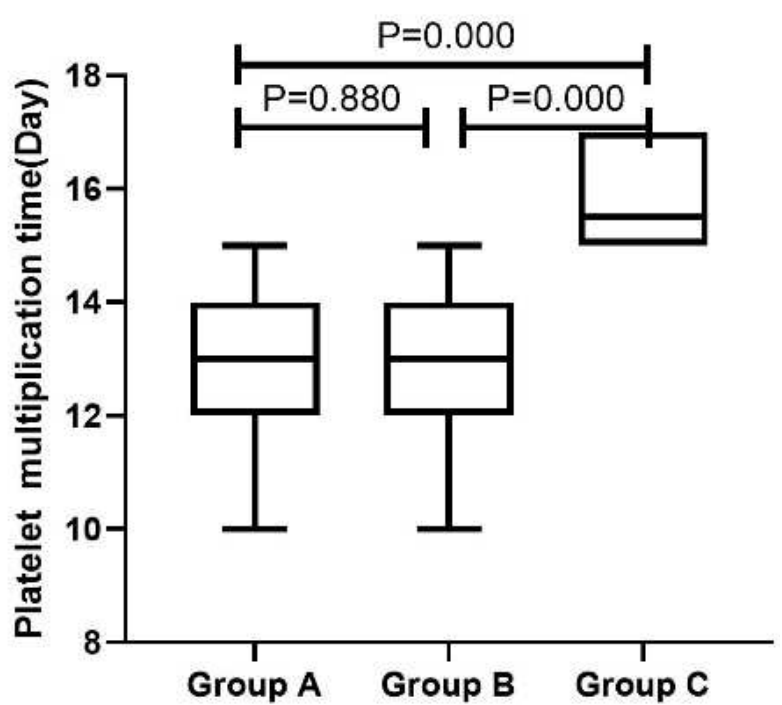


Table 1. Comparison of granulocyte and platelet graft time in each group (day $X \pm s)$.

\begin{tabular}{lll}
\hline Group & The transplantation time of granulocyte & the transplantation time of platelet \\
\hline Group A & $12.29 \pm 1.069$ & $12.71 \pm 1.326$ \\
Group B & $12.60 \pm 0.840$ & $12.80 \pm 1.390$ \\
Group C & $14.70 \pm 1.160$ & $15.80 \pm 0.919$ \\
\hline
\end{tabular}

Group A received three nursing interventions simultaneously; Group B received only one nursing interventions; Group C is the control group

\subsection{Comparison of Infection Probability Between Group A, $B$ and Control Group $C$}

The chi-square analysis showed that the incidence of respiratory and urinary tract infection could be effectively reduced when patients received three nursing interventions during the transplantation period $\left(\mathrm{X}^{2}=5.538, \quad \mathrm{X}^{2}=5.924\right.$; $P=0.019, P=0.05$; Table 2). Moreover, patients receiving only one of the nursing interventions can effectively reduce the incidence of respiratory and urinary tract infections. $\left(\mathrm{X}^{2}=5.609, \mathrm{X}^{2}=3.638 ; P=0.018, P=0.05\right.$; Table 3$)$. There was no statistical difference in the influence of nursing intervention on the incidence of digestive tract infection.

Table 2. Comparison of infection between Group A and control Group C (frequency).

\begin{tabular}{llll}
\hline Group & respiratory & digestive tract & urinary tract \\
\hline Group A & 1 & 0 & 1 \\
Group C & 6 & 2 & 5 \\
X2 & 5.538 & 0.997 & 5.924 \\
P & 0.019 & 0.318 & 0.05 \\
\hline
\end{tabular}

Group A received three nursing interventions simultaneously; Group C is the control group

Table 3. Comparison of infection between group B and control Group C (frequency).

\begin{tabular}{llll}
\hline Group & respiratory & digestive tract & urinary tract \\
\hline Group B & 2 & 1 & 2 \\
Group C & 6 & 2 & 5 \\
X2 & 5.609 & 0.882 & 3.638 \\
$\mathrm{P}$ & 0.018 & 0.350 & 0.05 \\
\hline
\end{tabular}

Group B received only one nursing interventions; Group C is the control group

\subsection{Comparison of Total Cost of Treatment Between Group $A, B$ and Control Group $C$}

The total cost of treatment for each group is shown in Table 4. What's more, further analysis found that the overall cost of patients receiving three nursing interventions was lower than those receiving no nursing intervention (A vs. C, $P=0.006$; figure 2). Even the total cost of patients receiving single nursing intervention was still lower than those who did not receive nursing intervention, although the difference was not statistically significant (B vs $\mathrm{C}, P=0.116$ ).

Table 4. Comparison of total cost of treatment during transplantation in each group (yuan $X \pm s$ ).

\begin{tabular}{ll}
\hline Group & Total cost of treatment during transplantation \\
\hline Group A & $16481 \pm 2393$ \\
Group B & $18171 \pm 3388$ \\
Group C & $21666 \pm 5765$ \\
\hline
\end{tabular}

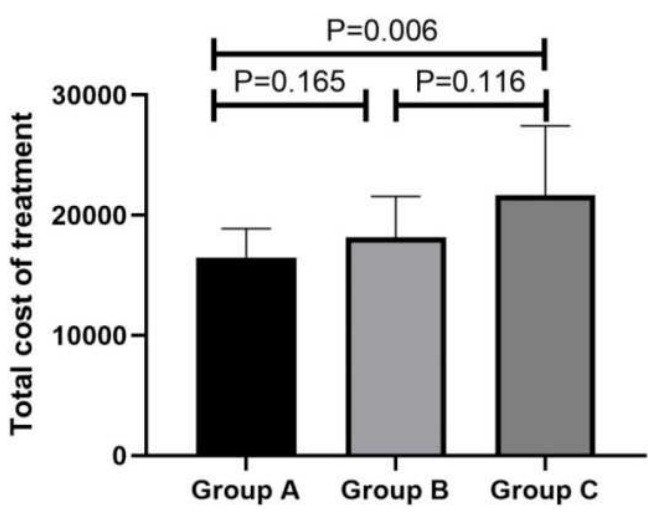

Figure 2. Comparison of total cost of treatment in each group.

\section{Discussion}

Allo-HSCT is an adoptive immunotherapy method to treat hematologic malignancies, bone marrow hematopoietic diseases and genetic metabolic disorders [7]. Allo-HSCT has become one of the rare methods to cure leukemia in clinical for those with high risk blood system disease [1, 3]. Before the implantation of stem cells into the receptor, a super-dose chemotherapy or systemic extensive radiotherapy is required to remove tumor cells, pathological hematopoietic cells, and immune cells from the body. This suggests that patients must experience a period of extreme immune deficiency prior to stem cell implantation survival and immune reconstitution.

Studies have shown that myelosuppression after transplantation is a period of significantly increased mortality. The most common cause of death was sepsis, which eventually led to septic shock, and the time of HSCT was positively correlated with infection rate [8]. The infection will be a fatal blow to the life and economy for patients with severe immune deficiency. In clinical practice, the traditional preventative treatment including systemic drug bath and negative pressure aseptic laminar flow ward monitoring, both have been widely accepted. However, the prophylactic use of antibiotics is still controversial [9]. In addition, predictive nursing is a new concept proposed in recent years, which refers to the simple way before the occurrence of adverse events to block the initiation of the event, so as to improve the prognosis of patients and reduce the cost of treatment [5]. Studies have shown that predictive care is effective in critically ill and surgical patients. However, the application and research of predictive nursing in patients with allo-HSCT during perioperative period is still lacking $[4,10]$.

In this study, clinical data of 36 patients with hematopathy who had undergone allo-HSCT were retrospectively analyzed. The results showed that predictive nursing intervention in the 
perioperative period of allo-HSCT could significantly shorten the transplantation time of granulocytes and megakaryocytes. Further analysis found that patients receiving oral care, perineal care and perianal care at the same time had a tendency to shorten the transplant time of stem cells compared with patients receiving only one of the nursing interventions. In addition, the analysis showed that the incidence of respiratory and urinary tract infection could be effectively reduced when patients received three nursing interventions during the transplantation period. Patients receiving only one of the nursing interventions can also effectively reduce the incidence of respiratory and urinary tract infections. The transplantation time of stem cell is shortened after predictive nursing intervention, which may be due to the low incidence of infection and Long-term intensive antibiotic therapy is not required in these patients. A large number of studies have shown that the restoration of intestinal microenvironment affects the viability of hematopoietic stem cells, and intensive antibiotic therapy is undoubtedly the most direct factor to destroy intestinal flora, which indirectly extends the implantation time of hematopoietic stem cell and the time needed for the reconstruction of immune function of patients [11-13]. What's more, analysis found that the overall cost of patients receiving three nursing interventions was lower than those receiving no nursing intervention. The total cost of patients receiving single nursing intervention was still lower than those who did not receive nursing intervention. Moreover, further analysis found that part of the higher costs came from expensive fortified antibiotic drugs, with antifungal drugs for lung infections accounting for the bulk of the cost, which was highly consistent with the high incidence of pulmonary infection during allo-HSCT [14].

\section{Conclusion}

In conclusion, the retrospective analysis found that various predictive nursing interventions including oral care, perineal care and perianal care in the perioperative period of HSCT could significantly shorten the transplantation time of granulocytes and megakaryocytes. On the other hand, the incidence of respiratory and urinary tract infection can be effectively reduced when patients received three nursing interventions during the transplantation period, even in those receiving only one type of care. What's more, analysis found that the overall cost of patients receiving three nursing interventions was lower than those receiving no nursing intervention. So predictive nursing is a cheap and effective method and worthy of clinical application. These results should prompt additional long-term studies to confirm.

\section{Acknowledgements}

Author Dongdong Chen and Jiaxiong Tan are the cocorresponding authors of this paper in no particular order. Due to they have made equally important contributions to the design, data collation and writing of this paper.

\section{References}

[1] Wang QY, Dong YJ, Liang ZY, Yin Y, Liu W, Xu WL, et al. [Clinical Analysis for Patients with AML Treated after Allo-HSCT]. Zhongguo Shi Yan Xue Ye Xue Za Zhi. 2020; 28 (4): 1105-1114.

[2] Zhu Y, Gao Q, Hu J, Liu X, Guan D, Zhang F. Allo-HSCT compared with immunosuppressive therapy for acquired aplastic anemia: a system review and meta-analysis. BMC Immunol. 2020; 21 (1): 10.

[3] Wang YF, Huang SY, Zhang ZH, Dong F, Wang Y, Jing HM, et al. [Clinical Efficacy of Allo-HSCT on FLT3-ITD Positive AML Patients]. Zhongguo Shi Yan Xue Ye Xue Za Zhi. 2020; 28 (4): 1183-1188.

[4] Heath LJ, Delate T, Weffald L, Paulson DC, Sanchez JK, Herner SJ. A Predictive Model to Identify Skilled Nursing Facility Residents for Pharmacist Intervention. Consult Pharm. 2018; 33 (9): 504-520.

[5] Meehan E, Foley T, Kelly C, Burgess Kelleher A, Sweeney C, Hally RM, et al. Advance Care Planning for Individuals With Chronic Obstructive Pulmonary Disease: A Scoping Review of the Literature. J Pain Symptom Manage. 2020; 59 (6): 1344-1361.

[6] Sturgess DJ, Greenland KB, Senthuran S, Ajvadi FA, van Zundert A, Irwin MG. Tracheal extubation of the adult intensive care patient with a predicted difficult airway - a narrative review. Anaesthesia. 2017; 72 (2): 248-261.

[7] Morris EC, Albert MH. Allogeneic HSCT in Adolescents and Young Adults With Primary Immunodeficiencies. Front Pediatr. 2019; 7: 437.

[8] Girmenia C, Bertaina A, Piciocchi A, Perruccio K, Algarotti A, Busca A, et al. Incidence, Risk Factors and Outcome of Pre-engraftment Gram-Negative Bacteremia After Allogeneic and Autologous Hematopoietic Stem Cell Transplantation: An Italian Prospective Multicenter Survey. Clin Infect Dis. 2017; 65 (11): 1884-1896.

[9] Lee SE, Lim JY, Ryu DB, Kim TW, Park SS, Jeon YW, et al. Alteration of the Intestinal Microbiota by Broad-Spectrum Antibiotic Use Correlates with the Occurrence of Intestinal Graft-versus-Host Disease. Biol Blood Marrow Transplant. 2019; 25 (10): 1933-1943.

[10] Barrett M, Boyne J, Brandts J, Brunner-La Rocca HP, De Maesschalck L, De Wit $\mathrm{K}$, et al. Artificial intelligence supported patient self-care in chronic heart failure: a paradigm shift from reactive to predictive, preventive and personalised care. EPMA J. 2019; 10 (4): 445-464.

[11] Staffas A, van den Brink M. The intestinal flora is required for post-transplant hematopoiesis in recipients of a hematopoietic stem cell transplantation. Bone Marrow Transplant. 2019; 54 (Suppl 2): 756-758.

[12] Hagan T, Cortese M, Rouphael N, Boudreau C, Linde C, Maddur MS, et al. Antibiotics-Driven Gut Microbiome Perturbation Alters Immunity to Vaccines in Humans. Cell. 2019; 178 (6): 1313-1328 e1313.

[13] Chen D, Wu J, Jin D, Wang B, Cao H. Fecal microbiota transplantation in cancer management: Current status and perspectives. Int J Cancer. 2019; 145 (8): 2021-2031. 
[14] Chauhan A, Gruenberg J, Arbefeville S, Mettler T, Brent CH,

Ferrieri P. Disseminated Hormographiella aspergillata Infection with Lung and Brain Involvement after Allogenic

Hematopoietic Stem-Cell Transplantation in a 54-Year-Old Man. Lab Med. 2019; 50 (4): 426-431. 